\title{
Industry 4.0 in maintenance: using condition monitoring in electric machines
}

\author{
Nuno Domingues \\ ISEL-ADEM \\ Rua Conselheiro Emídio Navarro, 1, 1959-007 Lisboa, Portugal \\ Email: nndomingues@gmail.com
}

\begin{abstract}
Industry traditionally considered maintenance as a cost and a necessity to replace equipment and machines, but the path has changed to better focus on maintenance to prevent faults and it was designated as predictive. The ones motivated to take these advantages are faced with two of the biggest barriers: the investment it requires and the difficulty to develop algorithms. The costs of installation are still high, but the avoided costs surpass it. Also, Internet of Things (IoT) has brought a big shift, which is been known as the industry 4.0. One of the potentials in maintenance is the conditioning monitoring. Condition monitoring sensors and devices are now linked to maintenance platforms, providing real-time data. This new connectivity is both more affordable and easier to implement than predictive maintenance. Real-time data allows managers to adjust preventive maintenance plans while providing greater reliability. At the same time, artificial intelligence manages this data to recognize patterns, which is one of the most promising advances in digital reliability. So, regardless of the ability to immediately implement a preventive maintenance plan, condition monitoring is an asset itself.
\end{abstract}

The present paper presents the common faults on electric machines, their effects, their impact on the industry and the main techniques on condition control to prevent them. It is also added the reflection on the use of IoT to enhance the potential of condition control maintenance.

The implementation of continuous improvement actions throughout the life of the equipment allows to increase efficiency, either by overcoming weaknesses or by adapting production or operational capacities to processes, production or maintenance, avoiding under maintenance or over maintenance and minimizing operating costs.

Index Terms - Condition monitoring, Condition control, damage detection, vibration analysis, model-updating

\section{INTRODUCTION}

$\mathrm{E}^{\mathrm{a}}$ quipment, machines, systems and facilities have evolved and modified over time, becoming increasingly sophisticated, requiring continuous improvement in maintenance processes and more rigorous dedication of the maintenance manager.

Despite the evolution in equipment and facilities, maintenance needs remain similar. For this reason, and understanding maintenance as a set of technical actions that allow to regulate the normal operation of these same equipment, one can divide it into three main types: (1) Corrective Maintenance, also known as reactive maintenance or "run until it breaks down "; (2) Preventive Maintenance, which is regular maintenance carried out according to defined schedules, regardless of the condition of the equipment; and (3) Predictive Maintenance, which is based on constant monitoring of operating equipment and forecasting failures. The condition-based maintenance is part of the Predictive Maintenance.

When an equipment breaks down, it must be repaired or replaced. This is the assumption of corrective maintenance, also called reactive maintenance. In other words, it is the technical activity performed after the occurrence of a malfunction and aims to restore the asset to a condition in which it can function as intended, either by repairing or replacing it. As this approach is characterized by maintenance actions after the occurrence of a failure, it is ideal for low priority equipment, without which the company's operations can continue to function normally. The same applies to less valuable equipment, as the work required to maintain it or constantly monitor it can end up being more expensive than repairing or replacing it when it breaks down. A simple example would be a light bulb, which can be used until it burns out and must then be replaced. As very little planning is needed for this approach, its implementation cost is very low compared to alternatives. The problem lies in relying on corrective maintenance for medium or high priority assets. Since no preventive actions are taken in a Corrective Maintenance strategy, the lifetime of the equipment will end up being shorter. If applied to high priority or value equipment, it will eventually lead to unexpected downtime and possibly huge repair costs. However, corrective maintenance is distinct from emergency maintenance: While corrective maintenance is carried out at a time when certain physical damage or disturbance in the normal operation of the equipment is evident (i.e., a functional failure), an emergency maintenance occurs after a total equipment failure, which requires urgent maintenance (and usually has higher costs).

Preventive maintenance appears as opposed to corrective maintenance: Rather than waiting for the breakdown to occur, this type of maintenance is aimed at preventing it from happening. Preventive maintenance takes place on a cyclical and scheduled basis, regardless of the asset's condition and with the objective of preventing damage and minimizing the 
consequences of equipment breakdown. The frequency is defined by the maintenance manager based on an estimate of the useful life of the equipment and the manufacturer's recommendations. Examples of preventive maintenance actions include periodic reviews, inspections, cleaning and lubrication of parts. This type of maintenance is essential for equipment essential for the normal operation of the company. In fact, the greater the risk associated with a given malfunction, the greater the need for preventive maintenance to increase the asset's useful life and reduce unplanned downtime. A classic example is elevators or freight elevators an elevator failure can be risky if someone gets trapped; the repair is lengthy and an out-of-service elevator is always extremely cumbersome. As they are not based on the actual condition of the equipment, preventive maintenance plans can sometimes be inefficient and result in unnecessary maintenance actions (including parts replacement) that cost time and money. The effect is compounded when a preventive approach is applied to low-priority or low-cost assets that could generate lower costs if only repaired on a reactive basis.

Even taking into account the potential waste of preventive maintenance, these expenses tend to be much lower than repairing the asset only when there is already a functional failure. Strategies focused on preventive maintenance represent savings in relation to corrective maintenance.

While there is a tendency to confuse reliability-centric maintenance with simple preventive maintenance, they are not the same thing. In short, the goal of reliability-centric maintenance is to increase asset availability. Obviously, this requires a big bet on preventive maintenance, but not only that. There are several types of maintenance that fit into a reliability-centric strategy, including predictive maintenance, which we'll focus on below. Therefore, while preventive maintenance and reliability-focused maintenance overlap, they do not completely overlap and should not be used synonymously.

Of all types of maintenance, predictive maintenance is the most recent and the one that requires the greatest technological investment. The goal of predictive maintenance is to predict when a malfunction is about to occur. When certain undesirable conditions are detected, then a repair is scheduled before the equipment actually malfunctions, thus eliminating the need for costly corrective maintenance or unnecessary preventive maintenance. It is based on the physical and operational condition of the equipment, through regular monitoring and testing of the condition and performance of the equipment, using advanced techniques such as vibration analysis, oil analysis, acoustics, infrared tests, or thermal imaging. This approach is based on the physical or operational condition of assets at the time of maintenance, rather than statistics and pre-defined calendars. It tries to detect the fault still in its hidden phase, before there is any visible sign, and in its potential phase. Thus, the maintenance performed will always be better informed, necessary and timely, since the equipment will only be subject to maintenance when a breakdown is foreseen, which will reduce costs and labor time spent on maintenance. The need to invest in specific monitoring equipment, as well as in the training of personnel to use them correctly and interpret the collected data, makes the implementation of this strategy very expensive, which is generally not within the reach of small and medium-sized companies. For this reason, it is not a profitable approach for assets that are not essential to the proper functioning of your operations.

Despite the high investment, predictive maintenance can represent big savings in the long term. Predictive maintenance is more effective at detecting potential malfunctions than preventive maintenance and is more incisive regarding what actions are actually needed.

\section{CONDITION CONTROL}

Predictive maintenance differs from condition-based maintenance, even though predictive maintenance also assesses the condition of each piece of equipment. Conditionbased maintenance focuses on well-defined analysis and parameters, defining an intervention to be made if detect something abnormal or there has been a clear change in the state of the equipment. Although predictive maintenance goes a step further by trying to detect failures at an even earlier stage.

The inspection plans for condition control techniques are designed according to the technical (exhaustive) characterization, the operational context of each equipment and articulated with the maintenance plans. Based on the information available in the context of action, via analysis of failures (equipment), failures (processes) and existing information on the architecture of equipment, actions are considered, defined and executed to improve processes.

Within the scope of control of the operational condition of equipment, inspection, measurement and diagnosis techniques allow accessing the state of the condition of each equipment in the operating context in which it is inserted and assess its condition by diagnosing abnormal situations, developing approaches to the analysis of breakdowns, or equating actions combined with the tasks defined in the maintenance plans. The use of inspection, measurement and diagnosis techniques allow access to the current state of the condition of each equipment and consider the operations to be carried out, together with the tasks defined in the maintenance plans. The plan has the following steps:

- Designs inspection and testing plans based on the technical characterization and operational context of each equipment and articulated with the maintenance plans.

- Performs inspection and condition control services, inspections in the fields of vibration analysis, ultrasound, thermography, electric current analysis, thickness measurements

- Assesses the condition of equipment in the operating context in which they operate, diagnosing abnormal situations and developing approaches for analyzing and limiting damages.

The main techniques are as follows:

- Inspections by analysis of vibrations, 
- Ultrasound inspections,

- Thermography inspections,

- Electric current analysis,

- Thickness measurements

Specialized interventions for dynamic balancing, on-site alignments are complemented by inspection plans and tests relating to inspections relating to assembly or repair processes or for receiving equipment via dynamic signature.

The implementation of continuous improvement actions throughout the life of the equipment allows to increase efficiency, either by overcoming weaknesses or by adapting production or operational capacities to processes, production or maintenance, avoiding undermaintenance or overmaintenance, minimizing operating costs and maintenance needs.

Tracking asset behavior is a big part of predictive maintenance - the data collected provides the foundation for finding trends and improving algorithms. It makes sense, however, to speak of real-time condition monitoring, even without a predictive maintenance program.

Advantages of monitoring the condition:

- Avoid major breakdowns and reduce downtime.

- Allows for better asset management throughout their lifetime.

- Lowers costs, especially with emergency maintenance.

- Provides the basis for developing predictive algorithms in the future.

In resume, condition monitoring consists of measuring equipment parameters that indicate failures. Ideally, it will be capable to detect changes in usual patterns in time to avoid a breakdown, save on corrective maintenance and prolong asset life. But one will only be able to enjoy these benefits if one chooses the correct techniques for monitoring the condition of the equipment and the operation.

\section{MAIN FAULTS IN AN INDUCTION MOTOR}

Currently, induction motors are the most used type of motor: this motor is widely used by industries due to its simple and robust structure, low costs, among other advantages. It is therefore necessary to ensure that the operation of these engines is carried out continuously and that it guarantees safety in their operation, as such it is necessary to study the main faults that may appear in an induction motor so that they are detected as soon as possible, thus avoiding expensive repairs or cut offs. These are some of the main malfunctions that can occur in an induction motor:

- Faults in the stator resulting from the opening or short circuit of one or more phases;

- Faults in the winding connections;

- Rupture of the rotor bars;

- Imbalances and mechanical misalignments;

- Irregularities in the air gap;

- Short circuit in the stator turns;

Some of these faults cannot be simulated in a Laboratory that all have access to, so one can only have a theoretical description of the detection of the problem.
Following are some failures that can take place in electric machines.

\section{A. Rotor failures}

Although it does not use any insulation, the rotor also suffers the consequences of an aging process. Each time a start is performed, on each bar, there are differential expansions between the lower and upper part because of the rotor current in the start, causing an uneven heating (higher on the upper part). On the other hand, different expansions from bar to bar may also appear due to an unequal distribution of currents between bars. These differences disappear once steady state operation is established and thermal equilibrium is reached. At each start-up, the rotor bars are also subjected to mechanical stresses, which, after many cycles, cause cracks and later complete failure of the bar due to mechanical fatigue. It is preferable to repair or replace the rotor of an important motor than to wait for its complete breakage, which normally leads to the destruction of the stator. It is therefore necessary to avoid any useless starting of electric motors.

\section{B. Loose rotor on shaft}

It is a malfunction resulting from a combination of a mechanical and electrical problem, arising when the rotor is loose from the shaft. In his spectral analysis, a wide band with a high level of vibration appears once the phenomenon occurs.

\section{Irregularities in the air gap}

Serious malfunction because if its detection is late, the machine may suffer serious damage. The air gap is the free space between the fixed (stator) and rotating (rotor) parts of a motor. This failure occurs when the rotor is not perfectly centered on the stator. The vibration caused by a rotor that is not centered on the stator takes place at a doble frequency equal (in Portugal it is $100 \mathrm{~Hz}$, since the grid frequency is 50 $\mathrm{Hz}$ ), regardless of the number of motor poles. Vibration amplitude increases with load and cancels out when motor power is turned off. This fact is due to some poorly tightened or warped engine support, which leads to deformation of the stator and an asymmetrical distribution of the air gap. One of the ways to detect the evolution of this fault is to analyze four air gap sensors, installed on the perimeter of the stator core. As the air gap is the smallest electromechanical pitch of the machine, the poles will serve as a physical reference for the entire axis of the machine. In other words, the sensors will grant us the distance between the stator core and the machine poles, in a certain time interval, through their own software.

\section{Dynamic eccentricity}

It arises when the rotor is not perfectly uniform despite being perfectly centered on the stator. This phenomenon occurs at 1xRPM and will disappear as soon as the motor power is cut off. This frequency will have sidebands at $2 \mathrm{xFd}$ (slip frequency).

\section{E. Breakage of rotor bars}

Rotor bar breakage is one of the most common malfunctions of squirrel cage induction motors. This type of 
malfunction does not immediately destroy the engine. The motor performance does not decrease, but the current increases in the bars adjacent to the bar where the break occurred, this usually causes an increase in temperature which, in turn, can lead to further damage. One or more broken rotor bars cause motor vibrations as those caused by dynamic eccentricity. The main difference is that the amplitude of the pole crossing frequency is proportional to the load, that is, this frequency will disappear when the motor is running with no load. Several studies prove that rotor bar breakage can be detected by looking at the stator current spectrum, where an additional line will appear at a frequency (1-2s). This spectral line is not easy to detect because it is very close to the fundamental current at frequency $f$. Therefore, a higher frequency, containing harmonics, is observed to detect the effect of bus breaks. This type of malfunction can result due to various factors such as thermal stresses due to overloads, uneven heat distribution, magnetic stresses due to electromagnetic forces, electromagnetic vibrations, deterioration of materials due to chemicals or moisture, etc.

\section{F. locked rotor}

Severe thermal deterioration in all phases of the winding usually caused by very high currents in the stator winding due to the locked or blocked condition of the rotor. This failure can also arise due to the excessive number of starts or reversals, incompatible with the normal speed for which the engine was designed.

\section{G. Stator faults}

Stator problems can generally be identified as one of the following causes: worn bearings, single-phase operation, moisture, poor insulation, overload, oil and debris. Factors that generally contribute to stator malfunctions are dust and debris. Some types of dust are highly conductive and help for insulation material failure. Ventilation restriction caused by dust on the motor temperature is the main reason for keeping the windings clean.

\section{H. Aging of insulators}

The life of the insulator varies proportionally with the increase in temperature. Thus, it can easily be concluded that any long-term overload that causes additional heating reduces the winding lifespan. It is recommended to regularly measure the current absorbed by the motor, preferably in each phase, and compare it with the value on the nameplate.

To avoid unwanted temperature increases in the motor, it is possible to mount thermal detectors in its winding that alert the user to this fact (alarm protections) or even cut off the motor power supply (trip protections). Another possibility, without the need to invest in the protection, would be to start monitoring the temperature of the motor housing. Being a simple and fast method, it allows to detect any heating in advance.

\section{Stator clearances}

Causes the appearance of $2 \mathrm{x}$ main frequency (in Portugal it is $100 \mathrm{~Hz}$, since the grid frequency is $50 \mathrm{~Hz}$ ), regardless of the number of poles. This phenomenon can still occur due to coiling or loose blades. This vibration will disappear as soon as the power to the electric motor is cut off.

\section{J. phase problems}

The detection of imbalances, in the currents absorbed by the three phases, in the order of two or three percent, may result from a faulty connection. In this case, we will have to check the tightness of all the terminal board females that tighten the power cables and the tightness of the cable terminal to the respective cable. In the frequency spectrum this problem will make visible the frequency of $2 x$ Frequency of the network (in Portugal it is $100 \mathrm{~Hz}$, since the grid frequency is $50 \mathrm{~Hz}$ ). Failure in a single phase of a winding is the result of an interruption of a motor supply phase. Lack of phase is usually caused by a blown fuse, lack of phase in the power line or poor contact due to damaged connections.

\section{K. Short circuit between phases}

This type of insulation failure is typically caused by winding contamination, vibration, or voltage surges. It can also be caused by the poor execution of insulation between phases, or even the use of inappropriate materials, incompatible with the thermal and voltage class of the equipment.

\section{Short circuit between turns}

This type of insulation failure is also caused, such as shortcircuiting phases, by contamination of the winding, vibration or voltage surges, which can be aggravated by failures or inefficiency of the impregnation process, including the application of conductors and varnish or poor-quality resin, incompatible with the thermal class and voltage of the equipment.

\section{Short circuit between blades}

This problem can occur in both the rotor and the stator. A short circuit between blades causes thermal deformation in the rotor and stator. Vibration increases after start-up, stabilizing later reaching operating temperature. Even with the rotor balancing, the short-circuited blades will cause an increase in the motor temperature, thus reducing its useful life.

\section{$N$. Failures in the winding connections}

The stator windings of an electrical machine are subject to several factors that can cause them to fail, such as thermal overloads, mechanical vibrations and voltage spikes. The study of fault detection and diagnosis in electrical machines, in particular in three-phase induction motors, requires practical experience in this field of knowledge. The threephase induction motor is one of the most used machines in the industry, all over the world. It is present in almost every type of industry, from small powers to high powers, from individual motors to motors integrated in electronic drives. Although this type of engine is very reliable, it is subject to a number of possible malfunctions. These breakdowns can become catastrophic and lead to production stoppages, physical damage or the unusability of raw material. The early 
detection of a malfunction, at its initial stage, can prevent the complete stoppage of a production line with the consequent gains in terms of production costs. Thus, it is very important to be able to diagnose an induction motor to detect possible faults in time.

\section{CONDITION-BASED MAINTENANCE IN ELECTRIC MACHINES}

The demand for electric machines has increased in the last decade, mainly due to applications that try to make a full transition from fuel to electricity. These applications encounter the need for tailor-made electric machines that must meet demanding requirements. Therefore, it is necessary for small-medium companies to adopt new technologies offering customized products fulfilling the customers' requirements according to their investment capacity, simplify their development process, and reduce computational time to achieve a feasible design in shorter periods. Furthermore, they must find ways to retain know-how that is typically kept within each designer to retrieve it or transfer it to new designers. [1] presents a framework with an implementation example of a knowledge-based engineering (KBE) system to design industrial electric machines to support this issue.

Magnetic flux analysis is a condition monitoring technique that is drawing the interest of many researchers and motor manufacturers. The great enhancements and reduction in the costs and dimensions of the required sensors, the development of advanced signal processing techniques that are suitable for flux data analysis, along with other inherent advantages provided by this technology are relevant aspects that have allowed the proliferation of flux-based techniques. This paper reviews the most recent scientific contributions related to the development and application of flux-based methods for the monitoring of rotating electric machines. Particularly, aspects related to the main sensors used to acquire magnetic flux signals as well as the leading signal processing and classification techniques are commented. The discussion is focused on the diagnosis of different types of faults in the most common rotating electric machines used in industry, namely: squirrel cage induction machines (SCIM), wound rotor induction machines (WRIM), permanent magnet machines (PMM) and wound field synchronous machines (WFSM) [2]

Performance of electric motors and losses in terms of heat and temperature are reviewed in this paper. Airgap eccentricity, electromagnetic performance, effect of temperature and losses are shown as factors affecting the efficiency. Several methods of computer aided analyses are listed. Temperature distribution in an induction motor is shown through the results of a simulation. [3]

Accurate temperature estimation of parts of electric machines using a lumped-parameter thermal network (LPTN) requires knowledge of power loss and thermal parameters. However, modeling parameter varying model inputs (power loss for each node and convective thermal conductances) based on physical equations using only measurement signals available during normal drive operation as inputs is not tangible. [4]

As the world is moving towards industry 4.0 standards, the problems of limited computational power and available memory are decreasing day by day. A significant amount of data with a variety of faulty conditions of electrical machines working under different environments can be handled remotely using cloud computation. Moreover, the mathematical models of electrical machines can be utilized for the training of AI algorithms. This is true because the collection of big data is a challenging task for the industry and laboratory because of related limited resources. [5]

Stator winding faults are one of the most common faults of permanent magnet synchronous motors (PMSMs), and searching for methods to efficiently detect this type of fault and at an early stage of damage is still an ongoing, important topic. This paper deals with the selected methods for detecting stator winding faults (short-circuits) of a permanent magnet synchronous motor, which are based on the analysis of the stator phase current signal. These methods were experimentally verified and their effectiveness was carefully compared. [6] presents the results of experimental studies obtained from the spectral analysis of the stator phase current, stator phase current envelope, and the discrete wavelet transform. The original fault indicators (FIs) based on the observation of the symptoms of stator winding fault were distinguished using the aforementioned methods, which clearly show which symptom is most sensitive to the incipient fault of the stator winding of PMSMs.

One of the most promising applications for high temperature superconducting (HTS) material is magnet. HTS magnet is considered to be used in a wide range of applications including MRI, particle accelerator, electrical machine, and etc. HTS rotating machines are mainly utilized for cryo-electrification, i.e. application of superconductivity for modern electrification. One of the main aspects of cryoelectrification is electric aircraft application which is recently enabled by continuous progress in design development of superconducting magnets and HTS machines.[7] discusses the challenges facing a newly developed magnet type, i.e. HTS ring magnet, that being considered in superconducting rotating machine in future electric aircrafts. HTS ring magnet is compact, easy to develop, fault tolerant, and light in weight, and it recently reached to a high level of magnetic field.

It is important to minimize the unscheduled downtime of machines caused by outages of machine components in highly automated production lines. Considering machine tools such as, grinding machines, the bearing inside of spindles is one of the most critical components. In the last decade, research has increasingly focused on fault detection of bearings. In addition, the rise of machine learning concepts has also intensified interest in this area. However, up to date, there is no single one-fits-all solution for predictive maintenance of bearings. Most research so far has only looked at individual bearing types at a time. [8] gives an overview of the most important approaches for bearing-fault analysis in grinding machines. There are two main parts of the analysis presented: The first part presents the classification of bearing faults, 
which includes the detection of unhealthy conditions, the position of the error (e.g. at the inner or at the outer ring of the bearing) and the severity, which detects the size of the fault. The second part presents the prediction of remaining useful life, which is important for estimating the productive use of a component before a potential failure, optimizing the replacement costs and minimizing downtime.

Condition-based maintenance starts with simple inspections. Small changes, such as abnormal heat or pressure, strange sounds, excessive vibration or a different smell, are often signs that something is going bad. However, condition monitoring techniques range from the simplest inspections to the most advanced technology. The main types of maintenance based on condition are as follows:

- Oil analysis applied to machine oils, lubricants and fluids. It can detect insulation, overheating and contamination. High levels of iron, e.g., usually indicate the presence of dust and sand. If detected early enough, gearbox failures can be reduced by $50 \%$. By avoiding contamination, bearing failure is also reduced by $75 \%$. To prevent these breakdowns, there are several oil analysis methods available: ferrography, water presence tests, viscosity tests, ICP/spectroscopy, dielectric strength test, microbial analysis, iron content (particle quantification index), infrared spectroscopy, ultraviolet spectroscopy, potentiometric titration (total number of acids/total number of bases) and sediment tests.

- Vibration analysis is one of the most popular methods of predictive maintenance. It can detect misalignments, imbalances and insulation for about 3 months before they cause a malfunction. It is also an opportunity to enhance energy consumption, since misaligned water pumps, e.g., use up to $15 \%$ more energy. Vibration measurement is also very prominent in the ISO 22096 standard. Vibration analysis condition monitoring techniques include shock pulse analysis (e.g., for rotating equipment), fast Fourier analysis, broadband vibration analysis (e.g., to detect bearing insulation), ultra- sonic (e.g., to detect leaks), power spectral density, time waveform analysis (e.g., for machines with XY probes) and spectrogram.

- Motor circuit analysis, also known as MCA testing, assesses the state of electric motors. It can be used to analyze motor condition (including rotors, coupling/belt problems, power quality), electrical imbalances and insulation. More than preventing failures, MCA can be used to reduce energy costs and improve equipment efficiency by $10-15 \%$.

- Thermography studies the patterns of heat and radiation in machines. Data analysis does the rest, detecting patterns that indicate failure or degradation. It has a wide range of applications, including detecting misalignments, imbalances, inadequate lubrication, insulation and tear on mechanical parts. In the electrical equipment, it identifies overheating, leaks in the pipes and weaknesses in the pressure tanks. Infrared thermography has become a popular method for predictive maintenance and non-destructive testing. Globally, thermography reduces risk, prevents failures, lowers costs and losses.

- Ultrasound Monitoring uses high-frequency sound waves to detect leaks and cavitations, which can reduce inspections by $30 \%$. Ultrasonic monitoring is especially cost-effective when done in conjunction with vibration analysis (some sensors pick up both) and infrared thermography. Aerial and structural ultrasounds are gaining traction as a good option for detecting 'stress waves' in rotating machines. Other methods include the backscatter technique and background wall echo attenuation.

- Radiography (including radiation analysis and neutron radiography), like thermography, is a very exhaustive method of non-destructive testing. Images allow technicians to inspect internal defects such as corrosion on bonded parts and weld failures. However, the main compared advantage is that it can be used in all types of materials.

- Laser interferometers measure changes to calculate displacements based on laser-generated wavelengths. It is used in condition monitoring to identify surface and subsurface defects such as pitting and pitting. Interferometry includes laser shear, laser ultrasound, strain mapping, electronic spot pattern interferometry, and digital holography.

- Electrical Monitoring. Is important to prevent accidental fires and injuries and fatalities at work. Preventing electrical failures with strict control not only prevents breakdowns but also improves safety. This includes tests to assess resistance, induction, electrical capacity, pulse response, frequency response, and degradation. Electrical condition monitoring techniques include megohmmeter tests, high potential or dielectric strength tests (e.g., to determine if the insulation is in good condition), energy signature analysis (to test current and voltage), impedance tests battery testing, overvoltage and undervoltage tests (also to detect insulation faults) and, to some extent, also motor circuit analysis.

- Electromagnetic monitoring measures magnetic field distortions to detect cracks, dents, corrosion, brittleness and other defects (e.g., insulation). Perhaps the most prominent method of monitoring the electromagnetic state is eddy current testing (ECT), which is used in the petrochemical industry to detect narrow cracks. In addition to ECT, there are other techniques such as pulsating eddy currents, distant and near-field eddy currents, low frequency saturated eddy current tests, eddy current matrix, magnetic particle inspection, magnetic flux leakage and metallic magnetic memory. These techniques seem especially suitable for nonferrous conductive materials such as tubes, condensers, boilers and aircraft surfaces. 


\section{CONCLUSION}

A conclusion section is not required. Although a conclusion may review the main points of the paper, do not replicate the abstract as the conclusion. A conclusion might elaborate on the importance of the work or suggest applications and extensions.

When first installed, the monitor learns the behavior of the electric motor to which it is connected. Spend some time learning before starting to monitor the engine.

Some electric motors drive equipment that operates at a constant speed and load. This is the simplest operating mode to learn and monitor, as any change in operating characteristics is likely indicative of a failure. Many machines operate at variable speed or variable load. In this case, what is normal for one load range may be abnormal for another. In this situation, the monitor learns and creates a separate internal engine model for each operating mode. Then, as conditions change, it changes from one model to another.

This cost-effective solution allows production facility owners to begin permanent monitoring of critical equipment with high ROI. Finally, it helps to reduce production downtime, reduce maintenance and repair costs, and increase the company's profitability.

Using condition monitoring techniques is the only way to fully understand the condition of electric machines and be able to carry out the right maintenance at the right time.

\section{REFERENCES}

[1] Rivera, C. A., Poza, J., Ugalde, G., \& Almandoz, G. (2021). Industrial Design of Electric Machines Supported with Knowledge-Based Engineering Systems. Applied Sciences, 11(1), 294.

[2] Zamudio-Ramirez, I., Osornio-Rios, R. A. A., Antonino-Daviu, J. A., Razik, H., \& de Jesus Romero-Troncoso, R. (2021). Magnetic Flux Analysis for the Condition Monitoring of Electric Machines: A Review. IEEE Transactions on Industrial Informatics.

[3] Gundabattini, E., Kuppan, R., Solomon, D. G., Kalam, A., Kothari, D P., \& Bakar, R. A. (2021). A review on methods of finding losses and cooling methods to increase efficiency of electric machines. Ain Shams Engineering Journal, 12(1), 497-505.

[4] Gedlu, E. G., Wallscheid, O., \& Böcker, J. (2021, May). Temperature estimation of electric machines using a hybrid model of feed-forward neural and low-order lumped-parameter thermal networks. In 2021 IEEE International Electric Machines \& Drives Conference (IEMDC) (pp. 18). IEEE.

[5] Kudelina, K., Vaimann, T., Asad, B., Rassõlkin, A., Kallaste, A., \& Demidova, G. (2021). Trends and Challenges in Intelligent Condition Monitoring of Electrical Machines Using Machine Learning. Applied Sciences, 11(6), 2761.

[6] Pietrzak, P., \& Wolkiewicz, M. (2021). Comparison of Selected Methods for the Stator Winding Condition Monitoring of a PMSM Using the Stator Phase Currents. Energies, 14(6), 1630.

[7] Yazdani-Asrami, M., Zhang, M., \& Yuan, W. (2021). Challenges for developing high temperature superconducting ring magnets for rotating electric machine applications in future electric aircrafts. Journal of Magnetism and Magnetic Materials, 522, 167543.

[8] Schwendemann, S., Amjad, Z., \& Sikora, A. (2021). A survey of machine-learning techniques for condition monitoring and predictive maintenance of bearings in grinding machines. Computers in Industry, 125,103380

\section{BIOGRAPHY}

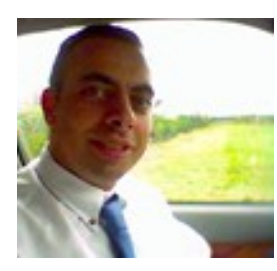

Nuno A. S. Domingues, Pós Doc in Science Communication from FCSH-UNL (2021), Pós Doc in Electrical Engineering and Computer Science from IST (2020), PhD in Electrical Engineering and Computer Science (in cooperation with Environmental Sciences Engineering) from FCT-UNL (2015), Master in Electrical Engineering and Computer Science from IST (2008), Undergraduate (5-year) degree in Electrical Engineering from ISEL (2005)

His topics of research include e-learning, science communication, education, electic machines, energy systems, electricity markets modelling and simulation, SCADA and DSS, decision making, intelligent optimization, evolutionary algorithms, machine learning, sustainability, efficiency, clean technologies, mobility and transport, sustainable consumption and regulation.

$\mathrm{He}$ is a Professor in ISEL. He holds a Problem Based Learning and Excelence on Teaching and Learning diploma by UNESCO and the Internationally Information Technology ECDL qualification. https://publons.com/researcher/1176435/nuno-domingues/metrics/ https://www.isel.pt/docentes/nuno-alexandre-soares-domingues https://orcid.org/0000-0003-0763-8106

https://scholar.google.pt/citations?user=-mtxKk8AAAAJ\&hl=pt-PT https://www.linkedin.com/in/nuno-domingues-a1401916/ 